\title{
Gate electrostatics and quantum capacitance in ballistic graphene device
}

Caridad, Jose; Power, Stephen R.; Shylau, Artsem; Gammelgaard, Lene; Jauho, Antti-Pekka; Bøggild, Peter

Published in:

Physical Review B (Condensed Matter and Materials Physics)

Link to article, DOI:

10.1103/PhysRevB.99.195408

Publication date:

2019

Document Version

Publisher's PDF, also known as Version of record

Link back to DTU Orbit

Citation (APA):

Caridad, J., Power, S. R., Shylau, A., Gammelgaard, L., Jauho, A-P., \& Bøggild, P. (2019). Gate electrostatics and quantum capacitance in ballistic graphene device. Physical Review $B$ (Condensed Matter and Materials Physics), 99(19), [195408]. https://doi.org/10.1103/PhysRevB.99.195408

\section{General rights}

Copyright and moral rights for the publications made accessible in the public portal are retained by the authors and/or other copyright owners and it is a condition of accessing publications that users recognise and abide by the legal requirements associated with these rights.

- Users may download and print one copy of any publication from the public portal for the purpose of private study or research.

- You may not further distribute the material or use it for any profit-making activity or commercial gain

- You may freely distribute the URL identifying the publication in the public portal 


\title{
Gate electrostatics and quantum capacitance in ballistic graphene devices
}

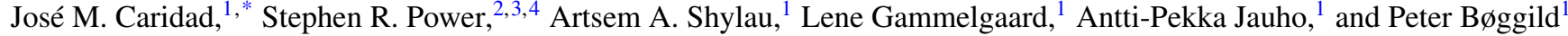 \\ ${ }^{1}$ Center for Nanostructured Graphene, Department of Physics, Technical University of Denmark, 2800 Kongens Lyngby, Denmark \\ ${ }^{2}$ Catalan Institute of Nanoscience and Nanotechnology (ICN2), CSIC and The Barcelona Institute of Science and Technology, \\ Campus UAB, Bellaterra, 08193 Barcelona, Spain \\ ${ }^{3}$ Universitat Autònoma de Barcelona, 08193 Bellaterra (Cerdanyola del Vallès), Spain \\ ${ }^{4}$ School of Physics, Trinity College Dublin, Dublin 2, Ireland
}

(Received 26 October 2018; published 6 May 2019)

\begin{abstract}
We experimentally investigate the charge induction mechanism across gated, narrow, ballistic graphene devices with different degrees of edge disorder. By using magnetoconductance measurements as the probing technique, we demonstrate that devices with large edge disorder exhibit a nearly homogeneous capacitance profile across the device channel, close to the case of an infinitely large graphene sheet. In contrast, devices with lower edge disorder $(<1 \mathrm{~nm}$ roughness) are strongly influenced by the fringing electrostatic field at graphene boundaries, in quantitative agreement with theoretical calculations for pristine systems. Specifically, devices with low edge disorder present a large effective capacitance variation across the device channel with a nontrivial, inhomogeneous profile due not only to classical electrostatics but also to quantum mechanical effects. We show that such quantum capacitance contribution, occurring due to the low density of states across the device in the presence of an external magnetic field, is considerably altered as a result of the gate electrostatics in the ballistic graphene device. Our conclusions can be extended to any two-dimensional (2D) electronic system confined by a hard-wall potential and are important for understanding the electronic structure and device applications of conducting 2D materials.
\end{abstract}

DOI: 10.1103/PhysRevB.99.195408

\section{INTRODUCTION}

An accurate picture of the electrostatic charge induction mechanism in field effect devices made of graphene or other two-dimensional (2D) crystals $[1,2]$ is necessary to understand the physical properties of these novel nanomaterials [1-18] as well as to guide their exploitation in novel electronic [18], spintronic [19], and optoelectronic [20] applications. Yet, the precise details of the gate electrostatics are far from being understood in these atomically thin and finite-size systems [4-10]. In general, the relation between the gate voltage $V_{g}$ and the induced average carrier density $n$ is complicated in 2D materials, arising not only from classical electrostatic interactions but also from quantum mechanical effects related to modifications in their band structure under gating [7-10]. The relation $V_{g}(n)$ is a function of the total capacitance per area of the system $C_{\text {tot }}$ and the elementary charge $e$ and can be expressed in terms of classical (electrostatic) $C_{c}$ and quantum capacitance $C_{q}$ contributions as $[8,9]$

$$
V_{g}=e n\left[C_{c}{ }^{-1}+C_{q}{ }^{-1}(n)\right]=e n C_{\text {tot }}^{-1}(n)
$$

where the dependence of $C_{q}$ on $n$ makes the capacitancevoltage relationship nonlinear in these systems $[8,9]$. Thus, $C_{\text {tot }}$ is a carrier density dependent property, sensitive to the device geometry, edge morphology and external measurement conditions such as the presence of a magnetic field $[9,10]$. Furthermore, it exhibits local variations since the charge carrier density $[n(\vec{r})]$ is not uniform in these finite-size systems:

*Corresponding author: jcar@dtu.dk
$C_{\mathrm{tot}}(\vec{r}) / e \equiv \alpha_{\mathrm{tot}}(\vec{r})=n(\vec{r}) \cdot V_{g}^{-1}[5-8]$. Here, $\vec{r}$ represents the coordinate(s) in which the carrier density varies and $\alpha_{\text {tot }}$ represents the so-called lever arm of the system.

We focus on the capacitance-voltage characteristics of high-quality, ballistic devices made of graphene, the frontrunner of the 2D materials [1,2]. While the injection of charge carriers in graphene field effect devices is often approximated by the infinite parallel-plate capacitor model $[1,2]$ where $n^{\infty}=\alpha_{c}^{\infty} V_{g}=\left(C_{\mathrm{ox}} / e\right) V_{g}$ and $C_{\mathrm{ox}}$ is the gate oxide capacitance per unit area, the presence of hard-wall boundaries leads to accumulation of charges close to the device edges as a consequence of the fringing electrostatic field [7,8] [Fig. 1(a)]. This results first in a generic, inhomogeneous, and position dependent effective capacitance profile of classical origin $\alpha_{c}(x)=C_{c}(x) / e$ across the device channel ( $x$ direction) which qualitatively follows a divergent $1 / \sqrt{x}$ dependence towards edges. Quantitatively, $\alpha_{c}(x)$ will depend on the entire device geometry, with the dominating factor being the ratio $W / b$ between sample width $W$ and the distance to the gate electrode $b: \alpha_{c}(x)$ increases for smaller $W / b$, especially for $W / b \leqslant 1$ [4,7-9]. Second, the presence of this varying $\alpha_{c}(x)$ results in a nontrivial potential profile $U(x)$ across these devices, a fact that may lead to the presence (or even dominance) of a quantum contribution $\alpha_{q}(x)=C_{q}(x) / e$ within the total effective capacitance $\alpha_{\mathrm{tot}}(x)=C_{\mathrm{tot}}(x) / e$ of the system [7-10].

Such complex gating dependencies are, however, largely unexplored and remain experimentally inconclusive. In particular, the existence of inhomogeneous capacitance profiles of classical origin $\alpha_{c}(x)=C_{c}(x) / e$ has been experimentally verified in high-quality, narrow graphene strips up to a certain modulation $\alpha_{\mathrm{tot}}(x)^{(\max )} / \alpha_{c}^{\infty}=C_{\mathrm{tot}}(x)^{(\max )} / C_{\mathrm{ox}}$ 

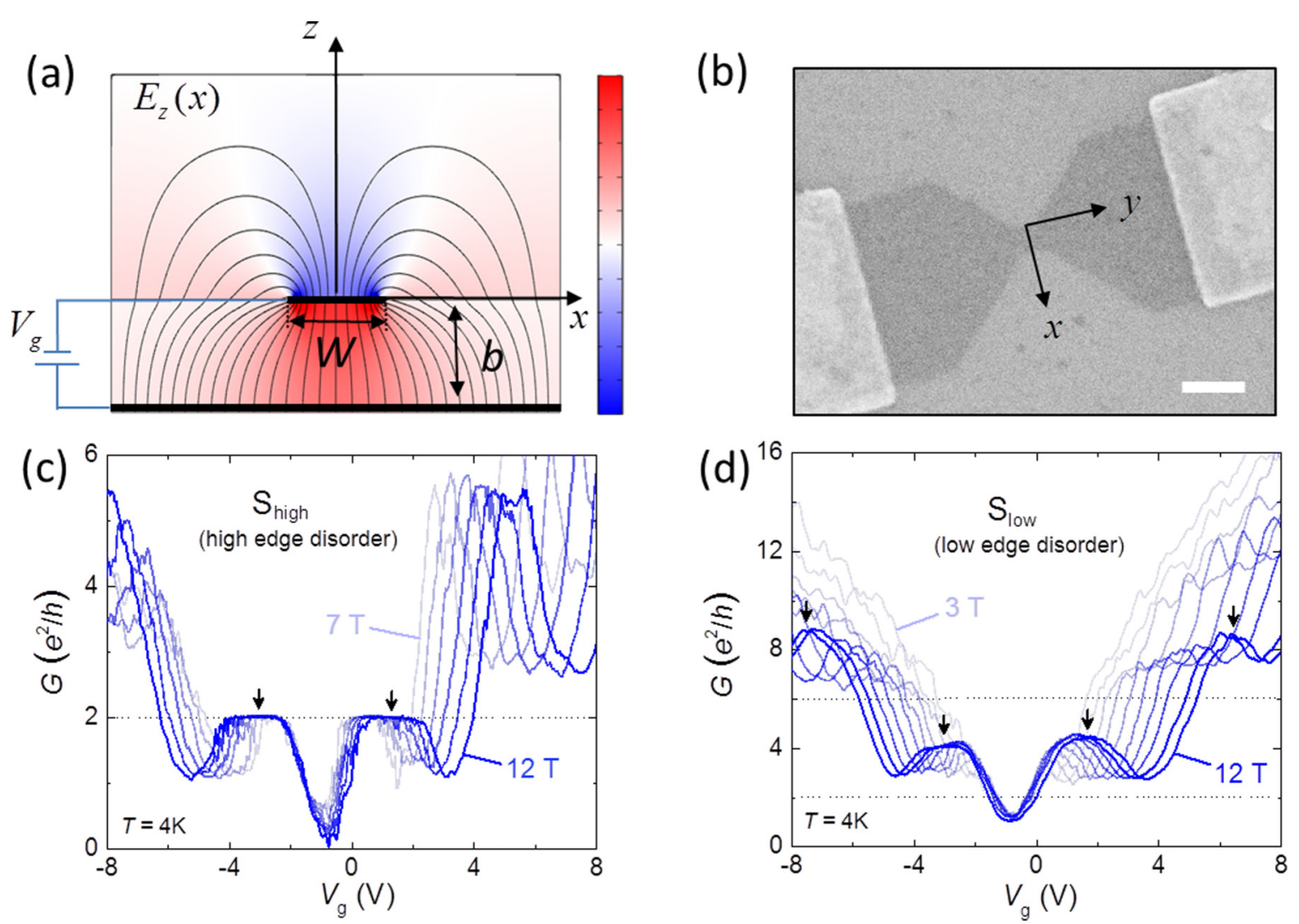

FIG. 1. Sample fabrication and electrical characterization. (a) Electric-field distribution $E_{z}(x)$ around a gated graphene device with $b=$ $W=100$ obtained by solving the Poisson equation in the device [27]. (b) Scanning electron micrograph of a graphene nanoconstriction device. Scale bar is $200 \mathrm{~nm}$. (c) Conductance $G$ vs gate voltage $V_{g}$ in a nanoconstriction of type $S_{\text {high }}$ at different magnetic fields (from 7 to $12 \mathrm{~T}$ in steps of $1 \mathrm{~T}$ ) at $T=4 \mathrm{~K}$. (d) Conductance $G$ vs gate voltage $V_{g}$ in a nanoconstriction of type $S_{\text {low }}$ at different magnetic fields (from 3 to $12 \mathrm{~T}$ in steps of $1 \mathrm{~T})$ at $T=4 \mathrm{~K}$. Arrows in panels (c) and (d) indicate the experimental points $\left(V_{g}, G\right)$ taken to calculate $\alpha_{v}\left(d_{v}\right)$ in Fig. 2 for $B=12 \mathrm{~T}$, i.e., the center of the plateau for sample $S_{\text {high }}[5,6]$ and $G^{\max }$ for $S_{\text {low }}$. Corresponding points are taken for other magnetic fields. Dotted lines indicate the expected quantization values for graphene.

[5], where $\alpha_{\text {tot }}(x)^{(\max )}$ is the maximum measured effective capacitance across the device. However, there are strong discrepancies between different experimental studies [3-6,11-17], and their correspondence with theoretical calculations, too [7-10]. Some studies exhibit capacitance profiles with modulation values across the device $\alpha_{\text {tot }}(x)^{(\max )} / \alpha_{c}^{\infty}$ $\sim 2$, close to those given by classical electrostatic predictions [5]; others report much smaller capacitance modulations $\left[\alpha_{\mathrm{tot}}(x)^{(\max )} / \alpha_{c}^{\infty}<1.2\right]$ across similar nanostructures [6], yet others observe no modulation at all $\left[\alpha(x)=\alpha_{c}^{\infty}\right]$ [3], or simply neglect the effect by assuming a constant effective capacitance [11-13]. In a wider perspective, transport studies carried out in high-quality graphene devices display variations in how inhomogeneous gating is accounted for, whether these effects are included $[4-6,14,15]$ or not at all $[3,11-13,16,17]$ during the interpretation of the electrical measurements.

Here, we probe the total effective capacitance profile $\alpha_{\text {tot }}(x)$ across ballistic graphene nanoconstrictions [Fig. 1(b)] with different degrees of edge disorder in a perpendicular magnetic field via magnetoconductance measurements. It is noteworthy that the local capacitance is accessible with these measurements in graphene devices [5,6,21], similar to conventional semiconductor structures defined in a $2 \mathrm{D}$ electron gas [5,22]. Indeed, magnetoconductance measurements are particularly relevant for the characterization of narrow $(\leqslant 150$ $\mathrm{nm}$ ) confined channels [22], where alternative magnetocapacitance techniques are limited due to several reasons. For instance, apart from possessing spatial resolution comparable to or larger than the device size, scanning gate microscopy [23], single-electron transistor [24], or microwave impedance spectroscopy $[14,25]$ measurements might affect or be affected by the actual device electrostatics. Specifically, by analyzing quantum Hall $(\mathrm{QH})$ transport measurements $[5,6]$ we show that devices with stronger edge disorder exhibit a nearly homogeneous effective capacitance profile, with $\alpha_{\text {tot }}(x)$ similar to $\alpha_{c}^{\infty}$. In striking contrast and in quantitative agreement with theoretical predictions for disorder-free systems, devices with a lower degree of edge disorder ( $<1 \mathrm{~nm}$ roughness) show an inhomogeneous effective capacitance consisting of both classical $\alpha_{c}(x)$ and quantum $\alpha_{q}(x)$ contributions. The presence of quantum capacitance effects in graphene devices is a direct consequence of the low density of states (DOS) of the system in a perpendicular magnetic field [10]. Also, $\alpha_{q}(x)$ is additionally influenced by band-structure modifications occurring in disorder-free, gated graphene devices-systems dominated by the electrostatic screening of the gate potential $[4,10]$. 
As shown below, the large $\alpha_{c}(x)$ diverging toward the edge is comparable in magnitude to or larger than $\alpha_{q}(x)$ at low carrier densities and certain positions $x$ across the channel of these clean devices, so that $\alpha_{q}(x)$ dominates the total effective capacitance [Eq. (1)].

\section{SAMPLE FABRICATION AND MAGNETOTRANSPORT MEASUREMENTS}

We have fabricated graphene nanoconstrictions [Fig. 1(b)] with similar lengths $L$ and widths $W$, $L=W \sim 100 \mathrm{~nm}$ on hydrophobized $\mathrm{SiO}_{2}$ substrates with thicknesses $b=100 \mathrm{~nm}$ and with different degrees of edge disorder [4]. Our initial graphene is exfoliated on hydrophobic $\mathrm{SiO}_{2}$, resulting in flakes with mobilities $\sim 20000 \mathrm{~cm}^{2} / \mathrm{Vs}$ [4,26] and mean free paths $l$ larger than $L, W$ at $T=4 \mathrm{~K}$ $(l \sim 200 \mathrm{~nm}[4,27])$. Therefore, the transport of carriers through these devices is limited by boundary scattering [4]. Furthermore, we note that the edge roughness in these samples can be precisely assessed via transmission electron microscopy [4], favoring their use in the present work with respect to nanoconstrictions [3] made from encapsulated graphene [28-30].

In particular, we study two types of nanoconstrictions, referred to as type $S_{\text {low }}$ and type $S_{\text {high }}$ made in an identical way except for the final etching step [4,31]. Sample type $S_{\text {low }}$ was etched using reactive ion etching (40 sccm argon, 5 sccm oxygen), a procedure producing significantly less edge disorder than oxygen plasma ashing $[4,31]$ technique used in sample type $S_{\text {high }}$. Further fabrication details of these devices can be found in [27] and in [4], where similar systems were studied. Measurements of differential magnetoconductance were performed at different magnetic fields using a Stanford SR830 lock-in amplifier with an excitation voltage of $100 \mu \mathrm{V}$ at a frequency of $17.77 \mathrm{~Hz}$ in a cryostat at $T=300$ and $4 \mathrm{~K}$.

Figures 1(c) and 1(d) show the magnetoconductance $G$ as a function of $V_{g}$ for the two sample types at different perpendicular magnetic fields $B$. The effect of edge disorder reflects itself in the magnitude of the conductance $G\left(V_{g}\right)$, which is more than two times smaller in type $S_{\text {high }}$ [Fig. 1(c)] compared to type $S_{\text {low }}$ [Fig. 1(d)] at any $B$ and $V_{g}$. Furthermore, sample type $S_{\text {high }}$ shows a quantized conductance $G=G\left(V_{g}\right)$ at high $B$ for the zeroth-order Landau level, LL0, at the corresponding filling factor for graphene: $v=2$. This plateau is followed by a conductance dip due to the presence of a nonzero longitudinal conductivity in the two-terminal device, effect that depends on the device geometry [4,32]. For the higher LLs, $G$ is smaller than the expected quantized value due to the pronounced effect of edge disorder [4], promoting backscattering between edge channels [33]. In contrast, as reported in our previous study [4], sample type $S_{\text {low }}$ (edge roughness $<1 \mathrm{~nm}$ ) shows a conductance the value of which is larger than the value expected for the single-electron picture and does not exhibit quantization. This effect, referred to as conductance quantization suppression (CQS) [4,34], is a manifestation of additional, overlapping, and counterpropagating conducting channels in the constriction. These channels emerge from a qualitative modification of the band structure of the system in the quantum Hall regime due to fringe field effects occurring in ballistic systems with low edge disorder [4,34] (see [27]). As such, their appearance is symptomatic of and relies on the presence of a large and inhomogeneous capacitance profile across the nanoconstrictions $[4,34]$.

\section{EFFECTIVE CAPACITANCE PROFILE}

We experimentally probe $\alpha_{\text {tot }}(x)$ in both types of samples $S_{\text {low }}$ and $S_{\text {high }}$ by using the evolution of the magnetotransport data shown in Figs. 1(c) and 1(d) with respect to different $B$ [the selected positions of $G\left(V_{g}, B\right)$ are marked by arrows]. This is possible thanks to the extreme sensitivity of the $\mathrm{QH}$ transport to the carrier density and carrier density distributions $[5,6]$. Briefly $[5,6]$, in the QH regime, when the Fermi level lies in-between two LLs and the conduction is governed by $v$ propagating edge states, each edge channel probes a different spatial region from the edge of the device [5], whereas the bulk is insulating $[5,6]$. On the one hand, the carrier density $n_{v}$ for a filling factor $v$ is given by $n_{v}=v e B h^{-1}$ with a corresponding effective capacitance $\alpha_{v}=n_{v}\left(V_{g}-V_{\mathrm{CNP}}\right)^{-1}$, where $V_{\mathrm{CNP}}$ is the charge neutrality point of the device. On the other hand, an estimate of the position of these edge channels is given by corresponding cyclotron diameter $d_{v}=$ $(\nu h)^{1 / 2}(e \pi B)^{-1 / 2}$ for graphene [5,6]. As the capacitance increase is linked to the charge accumulation across the channel of finite-size graphene devices, edge states of different LLs probe different spatial regions of the device and are subjected to a different effective capacitance. Thus, by making the assignment $\alpha_{v} \equiv \alpha\left(x=d_{v}\right)$, the profile of $\alpha_{\text {tot }}(x)$ across the device can be experimentally assessed [5,6]. Such capacitance mapping is clear in the absence of backscattering characteristic of the $\mathrm{QH}$ regime.

We argue that this semiclassical method can be also used in our ballistic devices exhibiting the CQS effect [sample type $S_{\text {low }}$, Fig. 1(d)]. This is particularly valid at gate voltages where the (nonquantized) conductance shows a maximum [arrows in Fig. 1(d)] $G^{(\max )}$, places where, if existent, backscattering between the counterpropagating edge channels in the system is weakest [4,34] (see [27]). As such, the link between $G$ and $n(x)$ is still valid by taking $v(x) \sim G^{(\max )}\left(e^{2} / h\right)$, and the capacitance profile $\alpha_{\text {tot }}(x)$ can then be extracted as demonstrated below when comparing the experimental values with the corresponding theoretical calculations.

Figure 2 shows the extracted effective capacitance $\alpha_{\nu}$ versus $d_{v}$ plotted for the different LLs in our samples with high and low edge disorder. Samples with high edge disorder [sample type $S_{\text {high }}$, Fig. 2(a)] show a slowly monotonically increasing capacitance $\alpha_{v}\left(d_{v}\right)$ towards edges, with quantitative values close to those of the infinite parallel-plate capacitor, $\alpha_{c}^{\infty}$ (green dotted line). Specifically, this capacitance profile is nearly homogeneous across the device channel ( $x$ direction), with only a small measured modulation up to $\alpha_{\text {tot }}(x)^{(\max )} / \alpha_{c}^{\infty} \sim 1.2$ for LLO. In contrast, devices with low edge disorder [sample type $S_{\text {low }}$, Fig. 2(b), ] exhibit an inhomogeneous capacitance profile, with much larger modulations $\left[\alpha_{\text {tot }}(x)^{(\max )} / \alpha_{c}^{\infty} \sim 2.5\right.$ for the case of LL0 and $\sim 1.7$ for LL1]. Moreover, we note how the capacitance profile is nonmonotonic in these samples for LLO. 

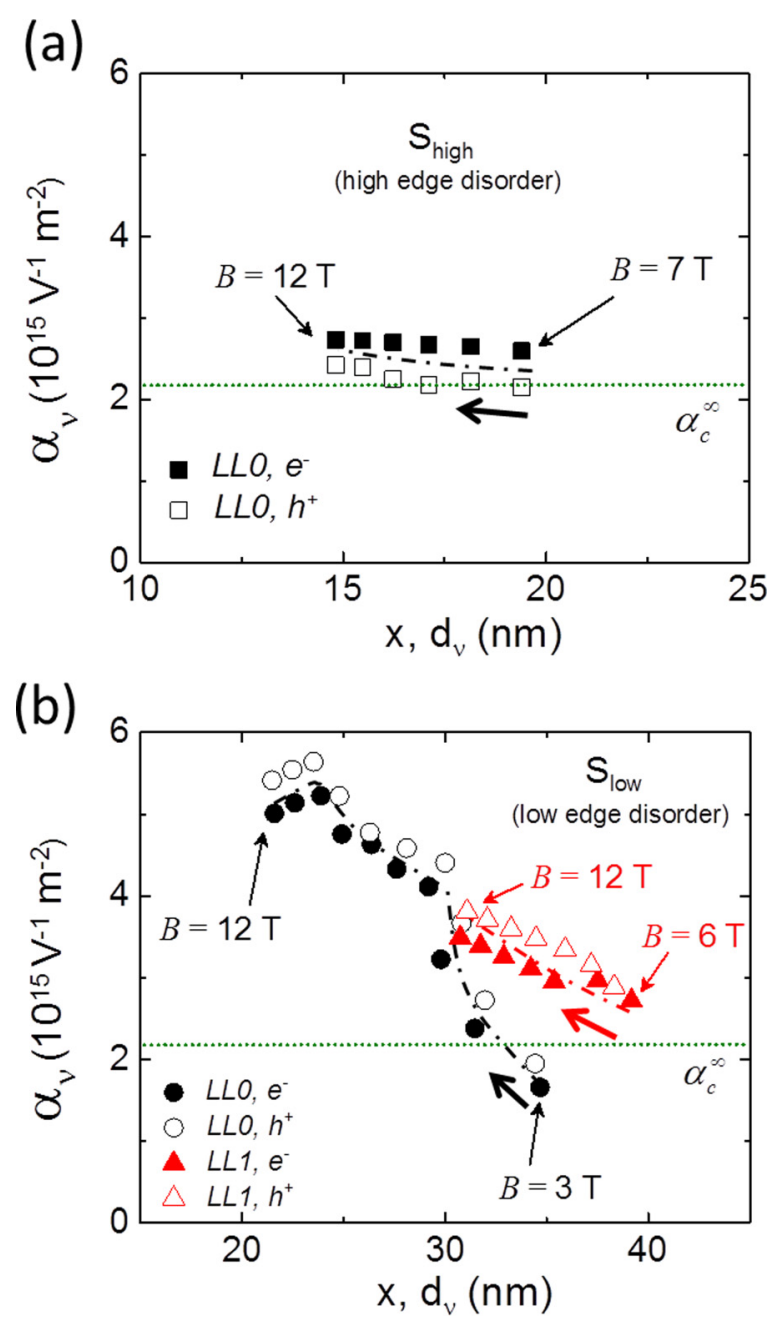

FIG. 2. Experimental effective capacitance $\alpha_{v}$ across the nanoconstrictions for different Landau levels (LLs) for electrons $\left(e^{-}\right)$ and holes $\left(h^{+}\right)$at positions $x$ corresponding to the cyclotron diameter $d_{v}$. (a) Sample type $S_{\text {high }}$. $B$ varies from 7 to $12 \mathrm{~T}$ increasing in 1-T steps. $B=7 \mathrm{~T}$ is the minimum $B$ to observe a conductance plateau for LL0 in samples $S_{\text {high }}$ (b) Sample type $S_{\text {low }} B$ varies from 3 to 12 $\mathrm{T}$ (LL0) and from $B=6$ to $12 \mathrm{~T}$ (LL1) in 1-T steps. $B=3$ and $6 \mathrm{~T}$ are the minimum fields to observe the CQS regime in these samples for LL0 and LL1, respectively. Green dotted lines in both panels show the capacitance value for an infinite parallel-plate capacitor $\alpha_{c}^{\infty}=\frac{C_{\mathrm{ox}}}{e}=2.19 \times 10^{15} \mathrm{~V}^{-1} \mathrm{~m}^{-2}$. Dash-dotted lines represent a guide to the eye.

Three initial conclusions can be drawn from the experimental data shown in Fig. 2, all of them consistent with a more pronounced capacitance profile for sample type $S_{\text {low }}$ as compared to $S_{\text {high }}$.

(i) As expected from an electrostatic point of view [6,35], there exists electron-hole $\left(e^{-}-h^{+}\right)$symmetry in both type of samples. The difference in capacitances observed for $e^{-}$and $h^{+}$is lower than $10 \%$ with respect to the absolute capacitance at any $B$.

(ii) The lowest LL (LL0) is closest to the edge, where more charge is expected to accumulate [4-8]. Consequently, for a constant $B, \alpha_{v}$ exhibits a higher absolute value for LLO than for LL1 in sample type $S_{\text {low }}$.

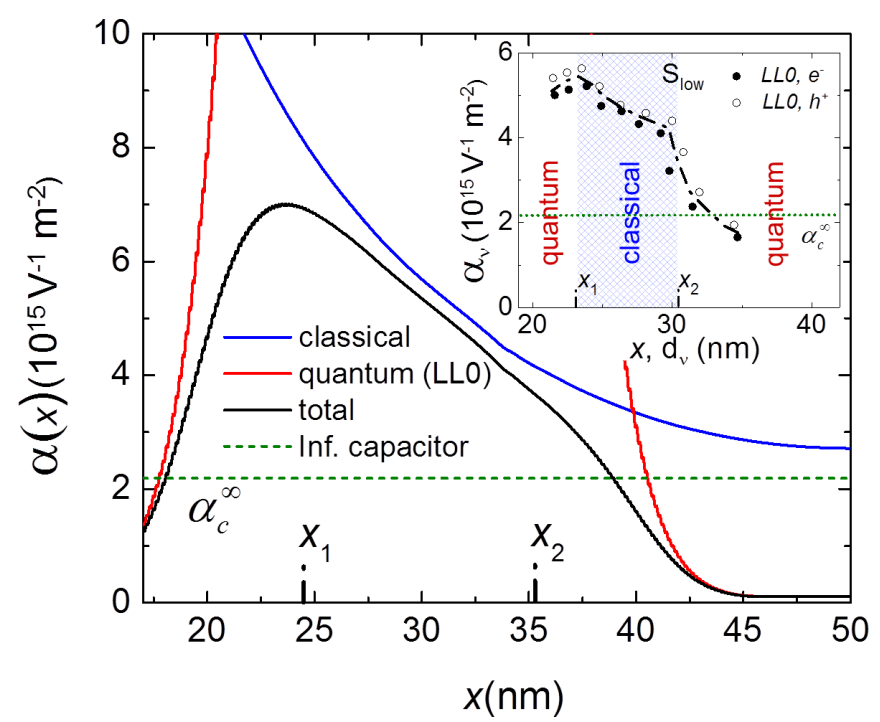

FIG. 3. Capacitance profile across the nanoconstrictions of type $S_{\text {low }}$ for LL0. Calculated classical (blue curve), quantum (red curve), and total effective capacitance (black curve) profiles across a graphene nanoconstriction with $W=100 \mathrm{~nm}$. The constant dashed green line represents $\alpha_{c}^{\infty}$. The inset shows the experimental data for sample type $S_{\text {low }}$ [Fig. 2(b)], separating zones where classical (blue) or quantum (red) contributions dominate according to the prediction shown in the main figure. Dash-dotted lines represent a guide to the eye.

(iii) For a varying $B$, the capacitance variation of LLO is the largest as well.

Next, we analyze in more detail the capacitance profile $\alpha_{v}\left(d_{v}\right)$ in both types of devices. For LLO, the small modulation $\alpha_{\text {tot }}(x)^{(\max )} / \alpha_{c}^{\infty} \sim 1.2$ in devices of sample type $S_{\text {high }}$ differs quantitatively from the electrostatic capacitance $\alpha_{c}(x)$ calculated for our constriction geometry, which is monotonic and diverges rapidly towards edges (Fig. 3, blue line, simulation details in [27]). Such behavior is attributed to scattering from edge defects, decreasing the charge accumulation in a similar manner as seen for low-quality devices with disorder in the channel [5,6]. This result explains the fact that some highquality devices in literature exhibit effective capacitances resembling the one from an infinite parallel-plate capacitor $\alpha_{c}^{\infty}[3,4]$.

Then, we note that despite having a large modulation, the capacitance variation in sample type $S_{\text {low }}$ does not completely follow the trend of the calculated classical electrostatic capacitance $\alpha_{c}(x)$ either: rather than following a divergent $1 / \sqrt{x}$ dependence towards the edge [7,8] the measured capacitance $\alpha_{v}$ has a nonmonotonic profile, decreasing more rapidly than $\alpha_{c}(x)$ for distances close to the edge and in the middle of the channel. Moreover, $\alpha_{v}$ exhibits some values smaller than $\alpha_{c}^{\infty}$ at the central part of the constriction (distances $>35 \mathrm{~nm}$ from the edge) for LL0 but not for LL1. In addition, $\alpha_{v}$ shows a maximum $\alpha_{v}^{(\max )}$ at a position $d_{\nu, \max } \sim 24 \mathrm{~nm}$ from the edge, with $\alpha_{v}$ smaller than $\alpha_{v}^{(\max )}$ for $d_{v}<d_{v \text {, max }}$. We do not expect this extremal value to be caused by the breakdown of the classical capacitance $\alpha_{c}(x)$ at distances from edges given by the magnetic length $l_{B}=\hbar^{1 / 2}(e B)^{-1 / 2}$ [7]: $d_{v, \max }$ is three times larger than $l_{B}$ at the corresponding $B(10 \mathrm{~T})$ 
at which the maximum is observed. This argument is additionally supported by the fact that no local maximum is experimentally observed in sample type $S_{\text {high }}$ [Fig. 2(a)] despite the minimum measured $d_{v}$ in such samples $(15 \mathrm{~nm})$ being smaller than $d_{v, \max }$.

\section{CAPACITANCE CALCULATION: CLASSICAL AND QUANTUM CONTRIBUTIONS}

We demonstrate that the mismatch between the calculated electrostatic capacitance (Fig. 3, blue line) and the experimentally probed capacitance of sample type $S_{\text {low }}$ at the LL0 [Fig. 2(b), black circular dots and Fig. 3, inset] can be fully accounted for by including quantum contributions to the total capacitance of the system. Such quantum contributions, arising due to the low local DOS [36-39] across the quasi-one-dimensional system [10], are not only dependent on the presence of external magnetic fields $B$ but also heavily affected by the electrostatic screening of the gate potential [4,34] (see [27]).

We calculate the quantum capacitance contribution [27] $\alpha_{q}(x)$ across the ballistic, gated devices at $B=10 \mathrm{~T}$ and low carrier densities (LL0) (Fig. 3, red line). First, we show that both classical and quantum contributions are comparable in magnitude and coincide at two distances: $\sim 25 \mathrm{~nm}$ far from the edge and at the central part of the nanodevice $(\sim 40 \mathrm{~nm}$ far from the edge). By using Eq. (1), we calculate the total effective capacitance $\alpha_{\text {tot }}(x)$ of these devices (Fig. 3, black curve), which is in quantitative agreement (below $\sim 30 \%$ mismatch) with our experimental data $\alpha_{v}\left(d_{v}\right)$ for LL0 [Fig. 2(b) and Fig. 3, inset]. Specifically, $\alpha_{\text {tot }}(x)$ shows a clear maximum $\alpha_{\text {tot }}^{(\max )}\left(x_{1}\right)$ at a position $x_{1} \sim 25 \mathrm{~nm}$, close to $d_{\nu, \max }$. For closer distances $\left(x<x_{1}\right)$ towards the edge at $x=0$, $\alpha_{\text {tot }}(x)$ is dominated by quantum contributions since $\alpha_{c}(x)>$ $\alpha_{q}(x)$. Instead, for distances $x_{1}<x<x_{2}$ with $x_{2} \sim 32 \mathrm{~nm}$, $\alpha_{\text {tot }}(x)$ is mostly dominated by the classical capacitance profile, since $\alpha_{c}(x)<\alpha_{q}(x)$ in this interval. Furthermore, for distances $x>x_{2}$ (central part of the nanoconstriction) $\alpha_{\text {tot }}(x)$ is again dominated by the quantum contribution due to the vanishing DOS at these positons. This argument explains the fact that, experimentally, $\alpha_{v}$ for LLO is smaller than $\alpha_{c}^{\infty}$ at the central part of the constriction, too. Finally, for completeness, we note that the experimental capacitance profile $\alpha_{v}$ shown for LL1 in samples $S_{\text {low }}$ [Fig. 2(b), red triangles] increases monotonically towards edges, similar to the classical profile. This behavior is due to the fact that the quantum contribution to the capacitance for LL1 is larger than the classical one [27] for the probed distances from the edge $x \equiv d_{v}$ (between 30 and $40 \mathrm{~nm}$ ). Thus, the classical contribution dominates the total effective capacitance profile at these higher carrier densities (LL1).

\section{CONCLUSIONS}

In summary, by analyzing quantum Hall transport measurements, we have extracted the total effective capacitance profile across ballistic graphene devices. Such profiles heavily depend on the edge disorder level in the device. In particular, similar to diffusive devices, ballistic samples with large edge roughness show a nearly homogeneous capacitance with small modulation and values close to the infinite parallelplate capacitor. In contrast, in excellent agreement with calculations for pristine and gated systems, narrow ballistic devices with low edge disorder $(<1 \mathrm{~nm}$ roughness) show an effective capacitance profile with a much larger modulation due to an enhanced impact of the fringe field effects at graphene edges. This profile is nonmonotonic at low carrier densities due to the interplay between classical and quantum capacitance contributions across the gated device even when using relatively thick $(100 \mathrm{~nm})$ dielectric layers [8,9]. Despite being demonstrated in ballistic graphene nanoconstrictions, our conclusions can be extended to other systems with sharp edge potential $[4,7,8,34]$, and, thus, our findings can help to understand the electronic properties of other types of gated nanostructures made from 2D materials [40,41] or other 2D systems [42].

\section{ACKNOWLEDGMENTS}

We acknowledge stimulating discussions with $K$. Kaasbjerg. This work was supported by the Danish National Research Foundation Center for Nanostructured Graphene (Project No. DNRF103) and the Union's Horizon 2020 research and innovation programme (Grant No. GrapheneCore2 785219). J.M.C. acknowledges funding from the $\varnothing$ tto Monsteds Fond. S.R.P. acknowledges funding from the European Union's Horizon 2020 research and innovation programme under Marie Skłodowska-Curie Grant No. 665919 and from the Irish Research Council under the laureate awards programme.
[1] K. S. Novoselov, A. K. Geim, S. V. Morozov, D. Jiang, Y. Zhang, S. V. Dubonos, I. V. Grigorieva, and A. A. Firsov, Electric field effect in atomically thin carbon films, Science 306, 666 (2004).

[2] Y. Zhang, Y. Tan, H. L. Stormer, and P. Kim, Experimental observation of the quantum Hall effect and Berry's phase in graphene, Nature (London) 438, 201 (2005).

[3] B. Terrés, L. A. Chizhova, F. Libisch, J. Peiro, D. Jörger, S. Engels, A. Girschik, K. Watanabe, T. Taniguchi, S. V. Rotkin, J. Burdörfer, and C. Stampfer, Size quantization of Dirac fermions in graphene constrictions, Nat. Commun. 7, 11528 (2016).
[4] J. M. Caridad, S. R. Power, M. R. Lotz, A. A. Shylau, J. D. Thomsen, L. Gammelgaard, T. J. Booth, A-P. Jauho, and P. Bøggild, Conductance quantization suppression in the quantum Hall regime, Nat. Commun. 9, 659 (2018).

[5] I. J. Vera-Marun, P. J. Zomer, A. Veligura, M. H. D. Guimarães, L. Visser, N. Tombros, H. J. van Elferen, U. Zeitler, and B. J. van Wees, Quantum Hall transport as probe of capacitance profile at graphene edges, Appl. Phys. Lett. 102, 013106 (2013).

[6] C. Barraud, T. Choi, P. Butti, I. Shorubalko, T. Taniguchi, K. Watanabe, T. Ihn, and K. Ensslin, Field effect in the quantum 
Hall regime of a high mobility graphene wire, J. Appl. Phys. 116, 073705 (2014).

[7] P. G. Silvestrov and K. B. Efetov, Charge accumulation at the boundaries of a graphene strip induced by a gate voltage: Electrostatic approach, Phys. Rev. B 77, 155436 (2008).

[8] J. Fernández-Rossier, J. J. Palacios, and L. Brey, Electronic structure of gated graphene and graphene nanoribbons, Phys. Rev. B 75, 205441 (2007).

[9] A. A. Shylau, J. W. Klos, and I. V. Zozoulenko, Capacitance of graphene nanoribbons, Phys. Rev. B 80, 205402 (2009).

[10] J. Guo, Y. Yoon, and Y. Ouyang, Gate electrostatics and quantum capacitance of graphene nanoribbons, Nano Lett. 7, 1935 (2007).

[11] K. I. Bolotin, K. J. Sikes, Z. Jiang, M. Klima, G. Fudenberg, J. Hone, P. Kim, and H. L. Stormer, Ultrahigh electron mobility in suspended graphene, Solid State Commun. 146, 351 (2008).

[12] X. Du, I. Skachko, A. Barker, and E. Y. Andrei, Approaching ballistic transport in suspended graphene, Nat. Nanotechnol. 3, 491 (2008).

[13] B. E. Feldman, J. Martin, and A. Yacoby, Broken-symmetry states and divergent resistance in suspended bilayer graphene, Nat. Phys. 5, 899 (2009).

[14] Y-T. Cui, B. Wen, E. Y. Ma, G. Diankov, Z. Han, F. Amet, T. Taniguchi, K. Watanabe, D. Goldhaber-Gordon, C. R. Dean, and Z.-X. Shen, Unconventional Correlation Between Quantum Hall Transport and Bulk State Filling in Gated Graphene Devices, Phys. Rev. Lett. 117, 186601 (2016).

[15] T. Taychatanapat, K. Watanabe, T. Taniguchi, and P. JarilloHerrero, Electrically tunable transverse magnetic focusing in graphene, Nat. Phys. 9, 225 (2013).

[16] H. Hettmansperger, F. Duerr, J. B. Oostinga, C. Gould, B. Trauzettel, and L. W. Molenkamp, Quantum Hall effect in narrow graphene ribbons, Phys. Rev. B 86, 195417 (2012).

[17] N. Tombros, A. Veligura, J. Junesh, M. H. D. Guimarães, I. J. Vera-Marun, H. T. Jonkman, and B. J. van Wees, Quantized conductance of a suspended graphene nanoconstriction, Nat. Phys. 7, 697 (2011).

[18] J. M. Caridad, G. Calogero, P. Pedrinazzi, J. E. Santos, A. Impellizzeri, T. Gunst, T. J. Booth, R. Sordan, P. Bøggild, and M. Brandbyge, A graphene-edge ferroelectric molecular switch, Nano Lett. 18, 4675 (2018)

[19] A. Dankert and S. P. Dash, Electrical gate control of spin current in van der Waals heterostructures at room temperature, Nat. Commun. 8, 16093 (2017).

[20] B. Yao, Y. Liu, S-W. Huang, C. Choi, Z. Xie, J. F. Flores, Y. Wu, M. Yu, D.-L. Kwong, Y. Huang, Y. Rao, X. Duan, and C. W. Wong, Broadband gate-tunable terahertz plasmons in graphene heterostructures, Nat. Photon. 12, 22 (2018).

[21] S. Ihnatsenka and I. V. Zozoulenko, Spin polarization and g-factor enhancement in graphene nanoribbons in a magnetic field, Phys. Rev. B 86, 155407 (2012).

[22] K. F. Berggren, G. Roos, and H. van Houten, Characterization of very narrow quasi-one- dimensional quantum channels, Phys. Rev. B 37, 10118 (1988).

[23] A. G. F. Garcia, M. Köning, D. Goldhaber-Gordon, and K. Tood, Scanning gate microscopy of localized states in wide graphene constrictions, Phys. Rev. B 87, 085446 (2013).

[24] J. Martin, N. Akerman, G. Ulbricth, T. Lohmann, K. von Klitzing, J. H. Smet, and A. Yacoby, Nat. Phys. 5, 699 (2009).
[25] E. Y. Ma, M. Reyes Calvo, J. Wang, B. Lian, M. Mühlbauer, C. Brüne, Y.-T. Cui, K. Lai, W. Kundhikanjana, Y. Yang, M. Baenninger, M. König, C. Ames, H. Buhmann, P. Leubner, L. W. Molenkamp, S.-C. Zhang, D. Goldhaber-Gordon, M. A. Kelly, and Z.-X. Shen, Unexpected edge conduction in mercury telluride quantum wells under broken time-reversal symmetry, Nat. Commun. 6, 7252 (2015).

[26] J. M. Caridad, S. Connaughton, C. Ott, H. B. Weber, and V. Krstić, An electrical analogy to Mie scattering, Nat. Commun. 7, 12894 (2016).

[27] See Supplemental Material at http://link.aps.org/supplemental/ 10.1103/PhysRevB.99.195408 for additional experimental data, discussion about the validity of the semiclassical method to probe capacitance profiles, and calculation of classical and quantum capacitances.

[28] L. Wang, I. Meric, P. Y. Huang, Q. Gao, Y. Gao, H. Tran, T. Taniguchi, K. Watanabe, L. M. Campos, D. A. Muller, J. Guo, P. Kim, J. Hone, K. L. Shepard, and C. R. Dean, One-dimensional electrical contact to a two-dimensional material, Science $\mathbf{3 4 2}$, 614 (2013).

[29] F. Pizzocchero, L. Gammelgaard, B. S. Jessen, J. M. Caridad, L. Wang, J. Hone, P. Bøggild, and T. J. Booth, The hot-pick up technique for batch assembly of van der Waals heterostructures, Nat. Commun. 7, 11894 (2016).

[30] P. Pedrinazzi, J. M. Caridad, D. M. A. Mackenzie, F. Pizzocchero, L. Gammelgaard, B. S. Jessen, R. Sordan, T. J. Booth, and P. Bøggild, High-quality graphene flakes exfoliated on a flat hydrophobic polymer, Appl. Phys. Lett. 3, 033101 (2018).

[31] P. Simonet, D. Bischoff, A. Moser, T. Ihn, and K. Ensslin, Graphene nanoribbons: Relevance of etching process, J. Appl. Phys. 117, 184303 (2015).

[32] J. R. Williams, D. A. Abanin, L. DiCarlo, L. S. Levito, and C. M. Marcus, Quantum Hall conductance of two-terminal graphene devices, Phys. Rev. B 80, 045408 (2009).

[33] H. Shen, A. Cresti, W. Escoffier, Y. Shi, X. Wang, and B. Raquet, Peculiar magnetotransport features of ultranarrow graphene nanoribbons under high magnetic field, ACS Nano 10, 1853 (2015).

[34] A. A. Shylau, I. V. Zozoulenko, H. Xu, and T. Heinzel, Generic suppression of conductance quantization of interacting electrons in graphene nanoribbons in a perpendicular magnetic field, Phys. Rev. B 82, 121410(R) (2010).

[35] S. Slizovskiy and V. Fal'ko, Suppressed compressibility of quantum Hall effect edge states in epitaxial graphene on $\mathrm{SiC}$, Phys. Rev. B 97, 075404 (2018).

[36] S. Dröscher, P. Roulleau, F. Molitor, P. Struderus, C. Stampfer, K. Ensslin, and T. Ihn, Quantum capacitance and density of states of graphene, Appl. Phys. Lett. 96, 152104 (2010).

[37] R. Reiter, U. Derra, S. Birner, B. Terrés, F. Libisch, J. Burgdörfer, and C. Stampfer, Negative quantum capacitance in graphene nanoribbons with lateral gates, Phys. Rev. B 89, 115406 (2014).

[38] J. Xia, F. Chen, J. Li, and N. Tao, Measurement of the quantum capacitance of graphene, Nat. Nanotechnol. 4, 505 (2009).

[39] G. L. Yu, R. Jalil, B. Belle, A. S. Mayorov, P. Blake, F. Schedin, S. V. Morozov, L. A. Ponomarenko, F. Chiappini, S. Wiedmann, U. Zeitle, M. I. Katsnelson, A. K. Geim, K. S. Novoselov, and D. C. Elias, Interaction phenomena in graphene seen through 
quantum capacitance, Proc. Natl. Acad. Sci. USA 110, 3282 (2013).

[40] K. S. Novoselov and A. H. Castro Neto, Two-dimensional crystal-based heterostructures: Materials with tailored properties, Phys. Scr. 2012, 014006 (2012).

[41] S. Rasappa, J. M. Caridad, L. Schulte, A. Cagliani, D. Borah, M. A. Morris, P. Bøggild, and S. Ndoni, High quality sub-
$10 \mathrm{~nm}$ graphene nanoribbons by on-chip PS- $b$-PDMS block copolymer lithography, RSC Adv. 5, 66711 (2015).

[42] T. Akiho, H. Irie, K. Onomitsu, and K. Muraki, Counterflowing edge current and its equilibration in quantum Hall devices with sharp edge potential: roles of incompressible strips and contact configuration, Phys. Rev. B 99, 121303(R) (2019). 